\title{
AN EXAMPLE OF A FUNCTION WITH NON-ANALYTIC ITERATES
}

\author{
M. LEWIN
}

(Received 11 January 1965)

\section{Preliminaries}

Let $\Omega$ be the set of the analytic functions $F(z)$, regular in some neighbourhood of the origin with the expansion

$$
F(z)=z+f_{2} z^{2}+f_{3} z^{3}+\cdots \cdot
$$

There may exist a function $F(s, z)$ analytic in $s$ and satisfying the following conditions ( $s$ and $s^{\prime}$ are any complex numbers):

$$
\begin{aligned}
& F(1, z)=F(z), \\
& F(s, z) \in \Omega, \\
& F\left[s, F\left(s^{\prime}, z\right)\right]=F\left[\left(s+s^{\prime}\right), z\right], \\
& F(s, z)=\sum_{k=1}^{\infty} f_{k}(s) z^{k} \text { for }|z|<\rho_{s},
\end{aligned}
$$

and the $f_{k}(s)$ are polynomials in $s$.

If $F(s, z)$ exists, it will be called the analytic iterate of $F(z)$. (The necessity and independence of these four conditions are discussed in [4]).

It is easily seen that $F(z)=z /(1-z)$ possesses the analytic iterate $F(s, z)=z /(1-s z)$. Functions which possess an analytic iterate will be called functions of type $A$. Functions for which (1.5) does not hold for every $s$, will be called functions of type $B$. In [2] I. N. Baker shows that for type $B$ the set $S$ of points $s$ for which (1.5) converges in some neighbourhood of $z=0$ is a discreet lattice (one- or two-dimensional). In [7] G. Szekeres shows that the class of entire functions of $\Omega$ belongs to $B$, also the class of rational functions of $\Omega$ unless $F(z)=z /(1-a z)$. Baker [3] extends the $B$-property to the class of meromorphic functions.

It is the purpose of this paper to prove the $B$-property for a certain function using much more elementary considerations. The function $e^{z}-1$ has been chosen to illustrate the method used. (By essentially the same method the functions $z+z^{2}$ and $z /(1-z)^{2}$ had been dealt with.) 


\section{Jabotinsky's $L$-functions}

Let $F(z) \in A$. It then has an analytic iterate $F(s, z)$. Define $L(z)$ by

$$
L(z)=\left.\frac{\partial F(s, z)}{\partial s}\right|_{s=0} .
$$

It is shown in [5] that the expansion of $L(z)$ is of the form

$$
L(z)=\sum_{k=\imath}^{\infty} l_{k} z^{k+l}, \text { for }|z|<\rho_{L}, \quad \rho_{L}>0,
$$

and that $L(z)$ satisfies the functional equation

$$
L[F(z)]=F^{\prime}(z) L(z),
$$

which may however have other solutions that are not $L$-functions of the function $F$. Clearly equation $(2.3)$ has the particular solution:

$$
L(z)=0 \text {, }
$$

whatever the given function $F$. The only function of $\Omega$ for which $L(z)=0$, is $F(z)=z$.

It is shown in [5] that the sequences $f_{n}$ in (1.1) and $l_{n}$ in (2.2) determine each other uniquely (though the series in (2.2) corresponding to a given function $F$ may converge only for $z=0$ ). To show that $F$ of $\Omega$ belongs to $B$ it is thus sufficient to show that the series (2.2) corresponding to this $F$ converges only for $z=0$.

$$
\text { 3. } F(x)=e^{x}-1
$$

Put $e^{z}-1=e^{x+i y}-1=u+i v(x, y, u, v$ real $)$, so that:

$$
u=e^{x} \cos y-1 ; v=e^{x} \sin y,
$$

and

$$
u^{2}+v^{2}=e^{2 x}-2 e^{x} \cos y+1
$$

It is easily seen that for $0<|y|<\pi / 2$ :

and for $y \neq 0$ :

$$
\frac{2(1-\cos y)}{\cos y}>y^{2}
$$

$$
2(1-\cos y)<y^{2}
$$

Lemma 3.1. The function $e^{z}-1$ maps each point of the right half-sirip $\operatorname{Re} z>0,-\pi<\operatorname{Im} z \leqq \pi$ either into the left half-plane (including the imaginary axis) or else farther away from the origin. 
PROOF. If $u \leqq 0$, there is nothing to prove. If

$$
u=e^{x} \cos y-1>0 \text {, }
$$

then

$$
\cos y>0 \text { and }|y|<\frac{\pi}{2}
$$

For $x>0$ :

$$
e^{x}-1>x,
$$

or, by squaring and subtracting $2 e^{x} \cos y$ from both sides:

$$
e^{2 x}-2 e^{x} \cos y+1>x^{2}+2 e^{x}(1-\cos y),
$$

But (3.5) implies:

$$
e^{x}>\frac{1}{\cos y}
$$

so that by (3.2), (3.8) and (3.9):

$$
u^{2}+v^{2}>x^{2}+\frac{2(1-\cos y)}{\cos y}>x^{2}+y^{2},
$$

the right inequality following from (3.3) and (3.6). This proves the lemma.

Lemma 3.2. The function $e^{z}-1$ maps all the points of the left half-strip $\operatorname{Re} z \leqq 0,-\pi<\operatorname{Im} z \leqq \pi$ nearer to the origin.

Proof. For $x=0$ this follows directly from (3.2) and (3.4). For $x<0$ :

$$
e^{x}<1,
$$

and also (since (3.7) holds generally for $x \neq 0$ )

$$
\left|e^{x}-1\right|<|x| \text {, }
$$

or

$$
e^{2 x}+1<x^{2}+2 e^{x}
$$

By squaring and subtracting as before we obtain $u^{2}+v^{2}<x^{2}+y^{2}$.

We now show the divergence of the $L(z)$ series. Equation (2.3) becomes in this case

$$
L\left(e^{z}-1\right)=e^{z} L(z) .
$$

It is sufficient to prove that this functional equation has no solution of the form (2.2) with a positive radius of convergence $\rho$, and which is not identically zero. 
It is obviously sufficient to consider the singular points of $L(z)$ in the strip $-\pi<\operatorname{Im} z \leqq \pi$ since $L(z)$ is periodic with period $2 \pi i$, if it is defined in a large enough region.

A. First suppose that $\rho$ is finite. $e^{z}-1$ maps the left half of the strip onto the circle $|z+1|<1$. If $\rho>0$ let $\zeta$ be a singular point of $L(z)$ on the circle of convergence. If $\zeta$ belongs to the left half-strip or is purely imaginary, then $w$ for which $w=e^{\zeta}-1$ is also a singular point and is nearer to the origin than $\zeta$ by lemma 3.2 which is a contradiction.

If $\zeta$ is a point of the right half-strip, then $\omega$ for which $e^{\omega}-1=\zeta$ is also a singular point. If $\zeta$ belongs to the right half-strip, then, by lemma 3.1, so does $\omega$ and also $|\omega|<|\zeta|$. Again there is a singular point inside the circle of convergence - a contradiction.

B. Suppose now that $L(z)$ is an entire function. It is then periodic and cannot therefore be a polynomial.

Equation (3.13) can be written in the form

$$
L(z)=(1+z) L[\log (1+z)] .
$$

This implies that for all large enough $r$ the function $M(r)=\max _{|z|=r}|L(z)|$ satisfies

$$
M(r)<2 r M(2 \log r),
$$

and putting, $V\left(e^{s}\right)=\log M(r), r=e^{s}$, the increasing function $V$ of $s$ satisfies $V(4 s)<V\left(e^{2}\right)<V(2 s)+s+\log 2$. Hence $(V(4 s)-V(2 s)) / 2 s<1$ for all large $s$ and thus it easily follows that $V(s)<s+K$ for some constant. Hence $V\left(e^{s}\right)<3 s+K$ so that

$$
M(r)<e^{K} r^{3},
$$

which implies $L(z)$ to be a constant $C$ which can only be zero because of (3.13). But $L(z)=0$ corresponds only to $F(z)=z$ so that our proof is complete.

\section{Acknowledgement}

The author wishes to thank Prof. Jabotinsky for his help in preparing a former version of this paper, and Prof. Baker for his many helpful remarks, which brought it into the present form.

\section{Bibliography}

[1] I. N. Baker, Zusammensetzungen ganzer Funktionen, Math. Z. 69 (1958), 121-163.

[2] I. N. Baker, Permutable power series and regular iteration, J. Australian Math. Soc. 2 (1962), 265-294.

[3] I. N. Baker, Fractional iteration near a fixpoint of multiplier 1., J. Australian Math. Soc. 4 (1964), 143-148. 
[4] P. Erdös and E. Jabotinsky, On analytic iteration, J. Analyse Math. 8 (1960-61), $361-376$.

[5] E. Jabotinsky, Analytic iteration, Trans. Amer. Math. Soc. 108 (1963), 457-477.

[6] G. Szekeres, Regular iteration of real and complex functions, Acta Math. 100 (1958), $203-258$.

[7] G. Szekeres, Fractional iteration of entire and rational functions, J. Australian Math. Soc. 4 (1964), 129-142.

The Technion, Israel Institute of Technology

Haifa, Israel 